\title{
Knee myopericytoma-case report and literature review
}

\begin{abstract}
Myopericytoma is a rare neoplasm, usually benign, presenting with histological differentiation towards perivascular myoid spindled cells/pericytes. Knee involvement is extremely rare, with only seven published cases.
\end{abstract}

The authors present the case of a patient with knee involvement myopericytoma, highlighting the diagnostic challenges particular to this location and review the previous cases reported in the literature.

Keywords: knee, myopericytoma, malignant myopericytoma
Volume 12 Issue 6 - 2020

\author{
Susana Rodrigues,' Catrine Ferreira, ${ }^{2}$ Tiago \\ Coelho,' Diogo Gaspar,' Jean Fallah,' Rui \\ Lino,' Nuno Geada' \\ 'Department of Orthopaedics and Traumatology, Garcia de \\ Orta Hospital, Portugal \\ 2Department of Pathology, Garcia de Orta Hospital, Portugal
}

Correspondence: Susana Rodrigues, Garcia de Orta Hospital, Department of Orthopaedics and Traumatology,Almada, Portugal, Tel 00351963732963,

Email susana.cadeias.rodrigues@gmail.com

Received: December 04, 2020 | Published: December 18, 2020

\section{Introduction}

Myopericytoma is an infrequent neoplasm, usually benign, presenting with histological differentiation towards perivascular myoid spindled cells/pericytes. ${ }^{1}$ This subset of pericytic tumors resulted from a recent distinction within a group of tumors, including myofibroma, infantile hemangiopericytoma, angioleiomyoma, and glomus tumor, formerly classified as hemangiopericytomas. ${ }^{1-3}$ Although, topographically, the lower limb is the preferred location for this tumor, knee involvement is extremely rare. ${ }^{4}$

Myopericytoma tend to be benign slow-growing tumors, yet, some cases can present with an invasive course with possibility of malignancy or progression to malignancy. ${ }^{5}$ The authors present the case of a patient with knee involvement myopericytoma, adjacent to the patellar tendon in an effort to highlight diagnostic challenges particular to this location.

\section{Case report}

A 73-year-old male presented with a chief complaint of left knee pain related to an antero-external mass. He reported knee pain for several years and that that the mass presented a couple of months ago. The patient noted recent increase in size and denied any or preceding trauma. Patient's history was unremarkable except for dyslipidemia and benign prostatic hyperplasia.

On physical examination, the mass was soft, round and mobile, with no overlying skin changes, tender and mildly painful to palpation.Ultrasound revealed a $6 \times 12 \mathrm{~mm}$-sized, well-defined, nodular formation with heterogeneous hypo echogenicity, adjacent to the patellar ligament.

Magnetic resonance imaging (MRI) revealed an ovoid, well circumscribed, mass with low signal intensity in T1 and high signal intensity in T2. Following gadolinium administration, it revealed diffuse and homogenous enhancement (Figure 1).

According to the mentioned clinical characteristics and following discussion with a sarcoma orthopedic surgeon expert, a decision was made to undergo an excisional biopsy.The surgical specimen consisted of a greyish mass well separated from the adjacent tissues and without vascular pedicle.

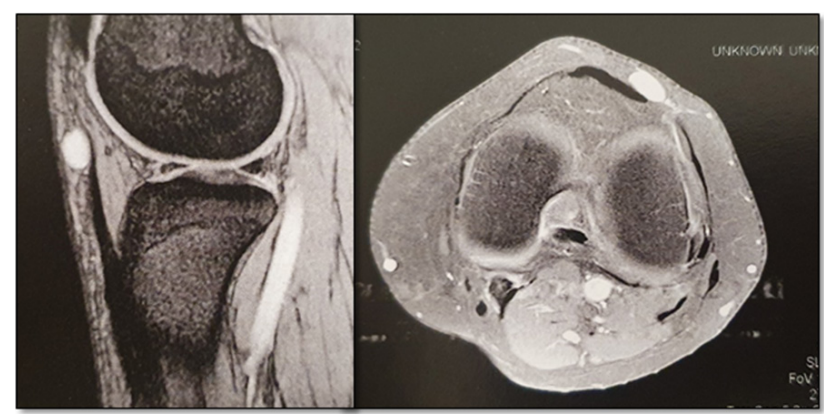

Figure I MRI revealing an ovoid, well circumscribed lesion, with diffuse and homogenous contrast enhancement.

Histologic examination revealed hematoxilin-eosin stains showing a well circumscribed nodular lesion, composed of oval-to spindleshaped myoid cells with a perivascular, multilayered and concentric growth.The neoplastic cells have eosinophilic cytoplasm and spindled nuclei (Figure 2). The histomorphological aspects were compatible with the diagnosis of myopericytoma.

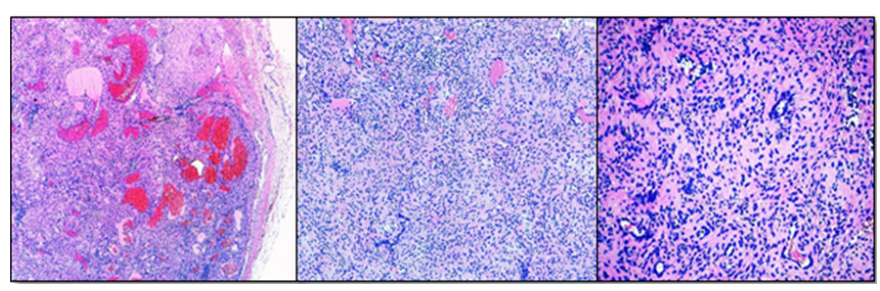

Figure 2 Histomorphological aspects compatible with the diagnosis of myopericytoma (hematoxilin-eosin stains).

The postoperative course was uneventful, with good wound healing and the patient was soon ambulating. Five months following surgery, the patient denied any complaints and had no evidence of local recurrence. ${ }^{6,7}$ 


\section{Discussion}

Mentzelet al. ${ }^{8}$ reported the largest myopericytoma series to date, in literature, with 54 cases. They found a 2:1 female:male predominance and a median age of 52 years ranging from 13 to 87 years. Regarding the knee region and to the best of our knowledge, only seven cases of myopericytoma have been previously published (Table 1).

Table I Knee myopericytoma cases in literature

\begin{tabular}{|c|c|c|c|c|}
\hline Study & Gender & Age & Size & Outcome \\
\hline Dray et al. ${ }^{7}$ & $M$ & 48 & $9 \mathrm{~mm}$ & No recurrence \\
\hline Dray et al. ${ }^{7}$ & $M$ & 36 & $45 \mathrm{~mm}$ & No recurrence \\
\hline Mentzel et al. ${ }^{8}$ & $M$ & 49 & - & No recurrence \\
\hline Mentzel et al..$^{8}$ & $\mathrm{~F}$ & 38 & - & No recurrence \\
\hline Mentzel et al. ${ }^{8}$ & $\mathrm{~F}$ & 39 & - & No recurrence \\
\hline Sohliya et al. ${ }^{6}$ & $\mathrm{~F}$ & 55 & $10 \mathrm{~mm}$ & No recurrence \\
\hline Choi et al. ${ }^{4}$ & $M$ & 61 & $12 \mathrm{~mm}$ & No recurrence \\
\hline
\end{tabular}

Unlike other locations, in knee myopericytoma, the gender distribution appears to be balanced. When interpreting this finding one must take in account the evident small sample.Our patient was considerable older than previous patients presenting with knee myopericytoma. We hypothesize that due to the benign slow-growing course of this tumor and lack of symptoms in initial stages, the diagnosis might have been deferred.

Anecdotal studies have reported that myopericytoma might be associated with trauma or immunocompromised states such as HIV infection. ${ }^{9}$ In this case there were no history of trauma neither immunodeficiency which corroborates with most of the cases being idiopathic in nature.Although myopericytoma tend to be a benign slow-growing tumor, some cases can present with an invasive course with potential for morbidity and mortality. Physicians should be alert to suspicious signs either clinic (rapid growth, adherence to adjacent tissues, overlying skin changes) or histologic (necrosis, pleomorphism and diffuse mitotic activity) as malignant myopericytoma may be easily overlooked. ${ }^{2,5}$

Surgical excision is the preferred approach in a subcutaneous growing mass, yet, close clinical control with imaging studies can be an option in selected cases. While the definitive diagnosis is histological, imaging studies are particularly important as the differential diagnosis must include neurogenic tumors and soft tissue sarcoma. Magnetic resonance imaging is considered the most useful in avoiding inadvertent excision of malignant soft tissue tumours. ${ }^{10}$

\section{Acknowledgments}

None.

\section{Conflicts of interest}

The authors declare there are no conflicts of interest.

\section{Funding}

None.

\section{References}

1. Granter SR, Badizadegan K, Fletcher CD. Myofibromatosis in adults, glomangiopericytoma, and myopericytoma: a spectrum of tumors showing perivascular myoid differentiation. Am J Surg Pathol. 1998;22(5):513525 .

2. McMenamin ME, Fletcher CD. Malignant myopericytoma: expanding the spectrum of tumours with myopericytic differentiation. Histopathology. 2002;41(5):450-460.

3. Jo VY, Fletcher CD. WHO classification of soft tissue tumours: an update based on the 2013 (4th) edition. Pathology. 2014;46(2):95-104.

4. Choi GW, Yang JH, Seo HS, et al. Myopericytoma around the knee: mimicking a neurogenic tumour. Knee Surg Sports Traumatol Arthrosc. 2016;24(9):2748-2751.

5. Binesh F, Moghadam RN, Shabani M, et al. Malignant Myopericytoma of Shoulder: A Rare Lesion. APSP J Case Rep. 2016;7(3):21.

6. Sohliya L, Mathew J, Ishitha G, et al. Myopericytoma-An Alternate Cause of Persistent Knee Pain in Rheumatoid Arthritis. J Assoc Physicians India. 2018;66(3):88-90.

7. Dray MS, McCarthy SW, Palmer AA, et al. Myopericytoma: a unifying term for a spectrum of tumours that show overlapping features with myofibroma. A review of 14 cases. J Clin Pathol. 2006;59(1):67-73.

8. Mentzel T, Dei Tos AP, Sapi Z, et al. Myopericytoma of skin and soft tissues: clinicopathologic and immunohistochemical study of 54 cases. Am J Surg Pathol. 2006;30(1):104-113.

9. Lau PP, Wong OK, Lui PC, et al. Myopericytoma in patients with AIDS : a new class of Epstein-Barr virus-associated tumor. Am J Surg Pathol. 2009;33(11):1666-1672.

10. Grimer R, Parry M, James S. Inadvertent excision of malignant soft tissue tumours. EFORT Open Rev. 2019;4(6): 321-329. 